\title{
A Frontier-Based Approach for Autonomous Exploration
}

\author{
Brian Yamauchi \\ Navy Center for Applied Research in Artificial Intelligence \\ Naval Research Laboratory \\ Washington, DC 20375-5337 \\ yamauchi@aic.nrl.navy.mil
}

\begin{abstract}
We introduce a new approach for exploration based on the concept of frontiers, regions on the boundary between open space and unexplored space. By moving to new frontiers, a mobile robot can extend its map into new territory until the entire environment has been explored. We describe a method for detecting frontiers in evidence grids and navigating to these frontiers. We also introduce a technique for minimizing specular reflections in evidence grids using laser-limited sonar. We have tested this approach with a real mobile robot, exploring real-world office environments cluttered with a variety of obstacles. An advantage of our approach is its ability to explore both large open spaces and narrow cluttered spaces, with walls and obstacles in arbitrary orientations.
\end{abstract}

\subsection{Introduction}

While many robots can navigate using maps, few can build their own maps. Usually a human must map the territory in advance, providing either the exact locations of obstacles (for metric maps) or a graph representing the connectivity between open regions (for topological maps). As a result, most mobile robots become unable to navigate efficiently when placed in unknown environments.

Exploration has the potential to free robots from this limitation. We define exploration to be the act of moving through an unknown environment while building a map that can be used for subsequent navigation. A good exploration strategy is one that generates a complete or nearly complete map in a reasonable amount of time.

Considerable work has been done in simulated exploration, but these simulations often view the world as a set of floorplans. The blueprint view of a typical office building presents a structure that seems simple and straightforward-rectangular offices, square conference rooms, straight hallways, and right angles everywhere-but the reality is often quite different. A real mobile robot may have to navigate through rooms cluttered with furniture, where walls may be hidden behind desks and bookshelves.

A few researchers have implemented exploration systems using real robots. These robots have performed well, but only within environments that satisfy certain restrictive assumptions. For example, some systems are limited to environments that can be explored using wall-following [6], while others require that all walls intersect at right angles and that these walls be unobstructed and visible to the robot [9]. Some indoor environments meet these requirements, but many do not.

Our goal is to develop exploration strategies for the complex environments typically found in real office buildings. Our approach is based on the detection of frontiers, regions on the border between space known to be open and unexplored space. In this paper, we describe how to detect frontiers in occupancy grids and how to use frontiers to guide exploration. Then we present results in which a real mobile robot used frontier-based exploration to map environments containing offices filled with furniture, hallways lined with obstacles, narrow passages, and large open spaces.

\subsection{Frontier-Based Exploration}

The central question in exploration is: Given what you know about the world, where should you move to gain as much new information as possible? Initially, you know nothing except what you can see from where you're standing. You want to build a map that describes as much of the world as possible, and you want to build this map as quickly as possible.

The central idea behind frontier-based exploration is: To gain the most new information about the world, move to the boundary between open space and uncharted territory.

Frontiers are regions on the boundary between open space and unexplored space. When a robot moves to a

Copyright 1997 IEEE. Published in the Proceedings of CIRA'97, July 10-11, 1997 in Monterey, California, USA. Personal use of this material is permitted. However, permission to reprint/republish this material for advertising or promotional purposes or for creating new collective works for resale or redistribution to servers or lists, or to reuse any copyrighted component of this work in other works, must be obtained from the IEEE. Contact: Manager, Copyrights and Permissions / IEEE Service Center / 445 Hoes Lane / P.O. Box 1331 / Piscataway, NJ 08855-1331, USA. Telephone: + Intl. 908-562-3966. 
frontier, it can see into unexplored space and add the new information to its map. As a result, the mapped territory expands, pushing back the boundary between the known and the unknown. By moving to successive frontiers, the robot can constantly increase its knowledge of the world. We call this strategy frontier-based exploration.

If a robot with a perfect map could navigate to a particular point in space, that point is considered accessible. All accessible space is contiguous, since a path must exist from the robot's initial position to every accessible point. Every such path will be at least partially in mapped territory, since the space around the robot's initial location is mapped at the start. Every path that is partially in unknown territory will cross a frontier. When the robot navigates to that frontier, it will incorporate more of the space covered by the path into mapped territory. If the robot does not incorporate the entire path at one time, then a new frontier will always exist further along the path, separating the known and unknown segments and providing a new destination for exploration.

In this way, a robot using frontier-based exploration will eventually explore all of the accessible space in the world-assuming perfect sensors and perfect motor control. A Zeno-like Paradox where the new information contributed by each new frontier decreases geometrically is theoretically possible (though highly unlikely), but even in such a case, the map will become arbitrary accurate in a finite amount of time.

The real question is how well frontier-based exploration will work using the noisy sensors and imperfect motor control of a real robot in the real world. This is the question that this research is intended to address.

\subsection{Laser-Limited Sonar}

We use evidence grids [7] as our spatial representation. Evidence grids are Cartesian grids containing cells, and each cell stores the probability that the corresponding region in space is occupied. Initially all of the cells are set to the prior probability of occupancy, which is a rough estimate of the overall probability that any given location will be occupied. Each time a sensor reading is obtained from the robot's sonar, infrared, or laser rangefinders, the corresponding sensor model is used to update the grid.

The main problem with sonar sensors is that instead of bouncing back toward the sensor, the sound pulse can hit a flat surface and bounce away from the sensor. Then either the sonar senses nothing, or it senses objects that, like reflections in a mirror, appear to be much farther away than the nearest surface.

These reflections could cause difficulties for frontierbased exploration, not only due to inaccuracies in the map, but also because specular reflections often appear as large open areas surrounded by unknown territory. As a result, the robot could waste a great deal of time trying to reach non-existent frontiers.

Fortunately, we have found a way to substantially reduce the effect of specular reflections on evidence grids. The standard evidence grid formulation assumes that each sensor reading is independent of every other sensor reading. In reality, this is not the case-and we take advantage of this with a technique we call laser-limited sonar. We use a laser rangefinder in combination with the sonar sensors, and if the laser returns a range reading less than the sonar reading, we update the evidence grid as if the sonar had returned the range indicated by the laser, in addition to marking the cells actually returned by the laser as occupied.

Why not just use the laser? Because the laser operates in a two-dimensional plane, while the sonar projects a three-dimensional cone. So, any object that is above or below the laser plane will be invisible to the laser, but still detectable by the sonar. Another alternative would be to use a three-dimensional laser rangefinder, but at present, such devices are too large, too expensive, and too powerhungry to be commonly available on mobile robots.

Laser-limited sonar isn't perfect-it is still possible to get specular reflections from obstacles undetected by the laser-but in practice, we have found that it drastically reduces the number of uncorrected specular reflections from walls and other large obstacles, which tend to be the major sources of errors in evidence grids built using sonar.

\subsection{Frontier Detection}

After an evidence grid has been constructed, each cell in the grid is classified by comparing its occupancy probability to the initial (prior) probability assigned to all cells. This algorithm is not particularly sensitive to the specific value of this prior probability. (A value of 0.5 was used in all of the experiments described in this paper.)

Each cell is placed into one of three classes:

- open: occupancy probability < prior probability
- unknown: occupancy probability = prior probability
- occupied: occupancy probability > prior probability

A process analogous to edge detection and region extraction in computer vision is used to find the boundaries between open space and unknown space. Any open cell adjacent to an unknown cell is labeled a frontier edge cell. Adjacent edge cells are grouped into frontier regions. Any frontier region above a certain minimum size (roughly the size of the robot) is considered a frontier. 


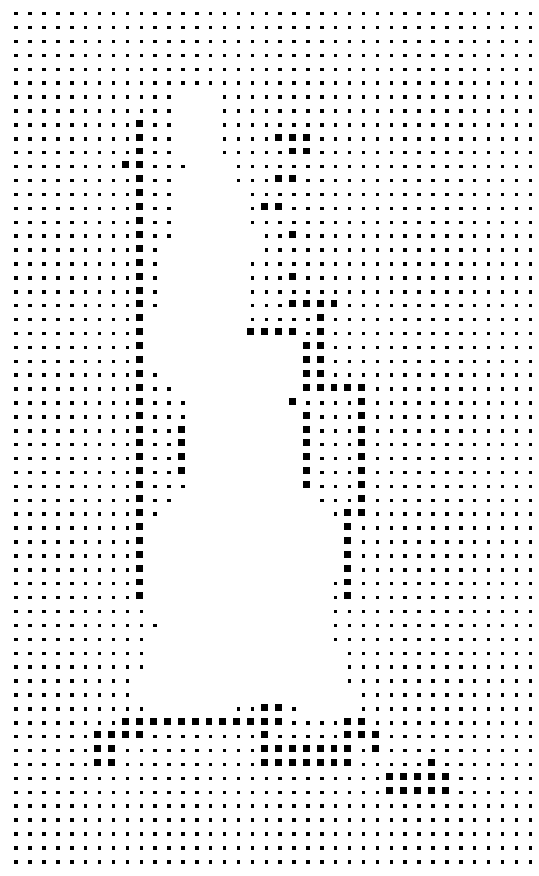

(a)

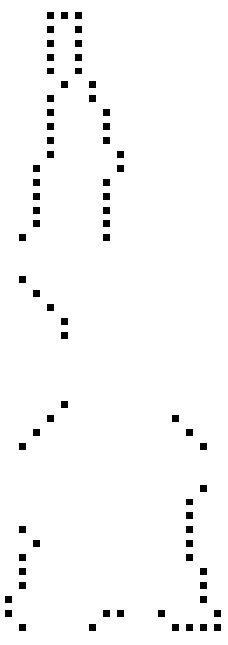

(b)
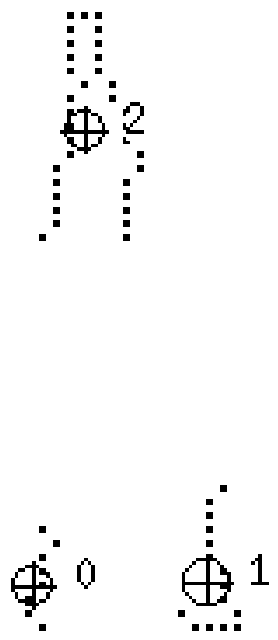

(c)

Figure 1: Frontier detection: (a) evidence grid, (b) frontier edge segments, (c) frontier regions

Figure 1a shows an evidence grid built by a real robot in a hallway adjacent to two open doors. Figure $1 \mathrm{~b}$ shows the frontier edge segments detected in the grid. Figure 1c shows the regions that are larger than the minimum frontier size. The centroid of each region is marked by crosshairs. Frontier 0 and frontier 1 correspond to open doorways, while frontier 2 is the unexplored hallway.

\subsection{Navigating to Frontiers}

Once frontiers have been detected within a particular evidence grid, the robot attempts to navigate to the nearest accessible, unvisited frontier. The path planner uses a depth-first search on the grid, starting at the robot's current cell and attempting to take the shortest obstacle-free path to the cell containing the goal location.

While the robot moves toward its destination, reactive obstacle avoidance behaviors prevent collisions with any obstacles not present while the evidence grid was constructed. In a dynamic environment, this is necessary to avoid collisions with, for example, people who are walking about. These behaviors allow the robot to steer around these obstacles and, as long as the world has not changed too drastically, return to follow its path to the destination.
When the robot reaches its destination, that location is added to the list of previously visited frontiers. The robot performs a 360 degree sensor sweep using laser-limited sonar and adds the new information to the evidence grid. Then the robot detects frontiers present in the updated grid and attempts to navigate to the nearest accessible, unvisited frontier.

If the robot is unable to make progress toward its destination, then after a certain amount of time, the robot will determine that the destination in inaccessible, and its location will be added to the list of inaccessible frontiers. The robot will then conduct a sensor sweep, update the evidence grid, and attempt to navigate to the closest remaining accessible, unvisited frontier.

\subsection{Experiments}

Frontier-based exploration has been implemented on a Nomad 200 mobile robot equipped with a laser rangefinder, sixteen sonar sensors, and sixteen infrared sensors. Laser-limited sonar is used to build evidence grids, combining the data from the forward-facing sonar with the data returned from the (forward-facing) laser rangefinder. At extremely short ranges (less than 16 
inches), range data from the infrared sensor is used instead. All sixteen sonar and infrared sensors are used for obstacle avoidance. All computation for frontier-based exploration is performed by an offboard Sparcstation 20. The process running on the workstation communicates with the robot's onboard 486 processor via a radio ethernet.

We have conducted experiments using frontier-based exploration in two different real-world office environ- ments. The first environment included a hallway and an adjacent office. This environment contained chairs, desks, tables, bookcases, filing cabinets, a sofa, a water cooler, and boxes of varying size and shape.

Figure 2 shows the results from a trial in which the robot started in the hallway, used frontier-based exploration to detect and enter an open doorway, and then explored the adjacent office ( 23 feet x 20 feet) thoroughly.

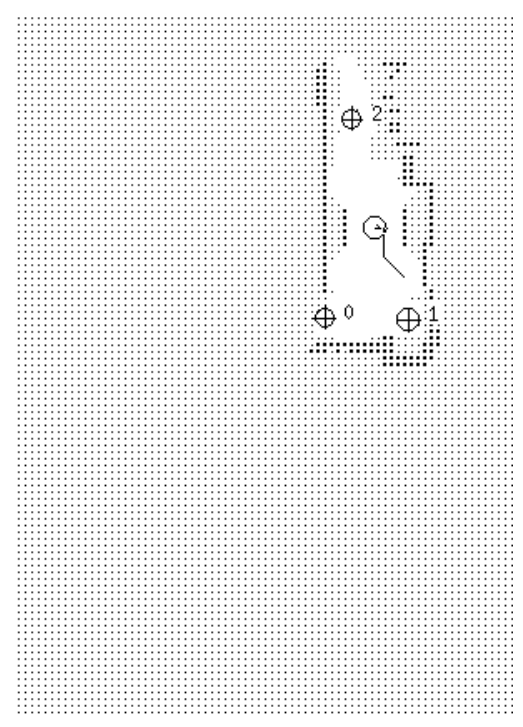

(a)

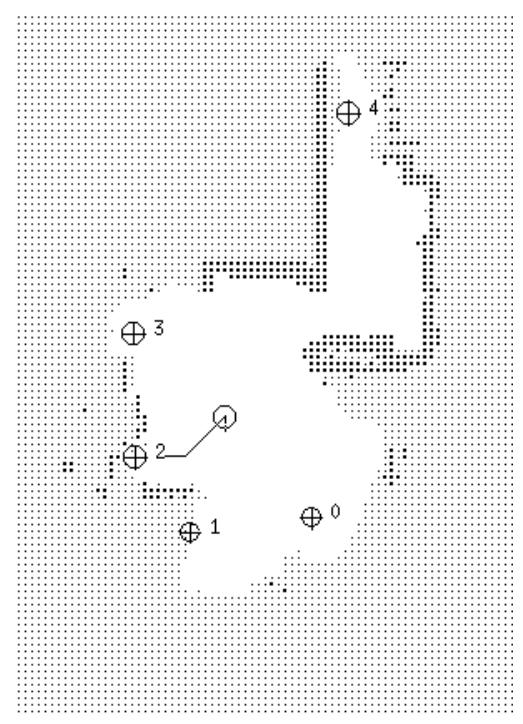

(d)

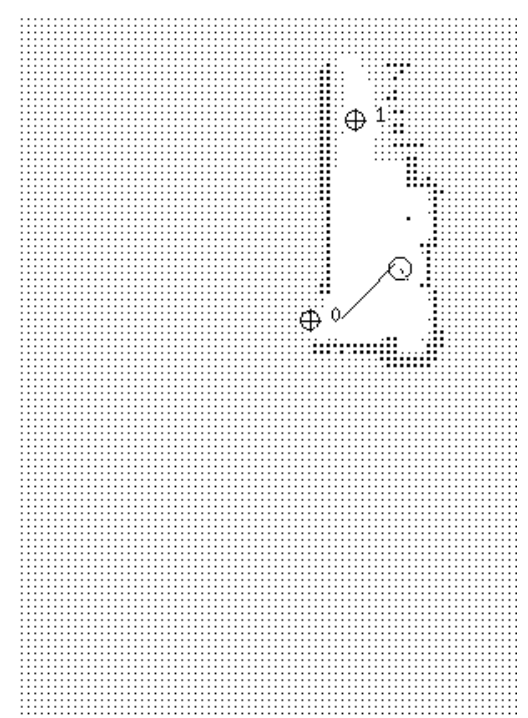

(b)

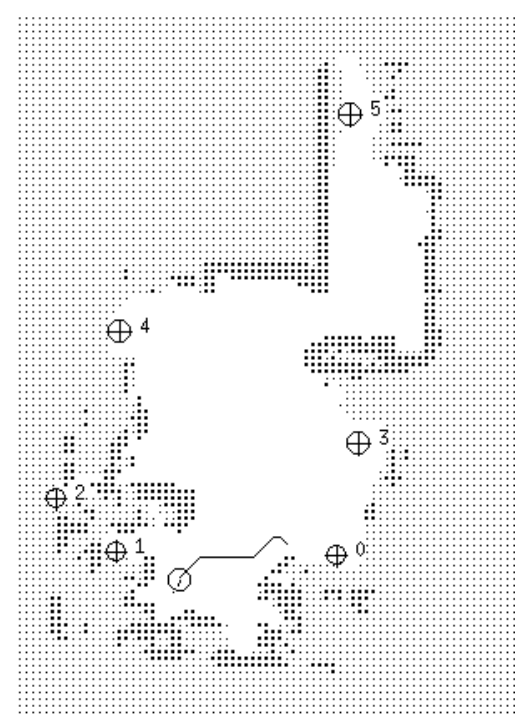

(e)

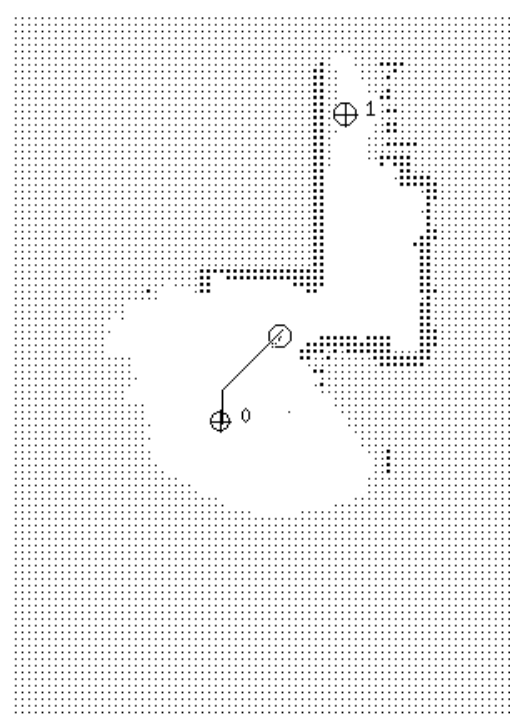

(c)

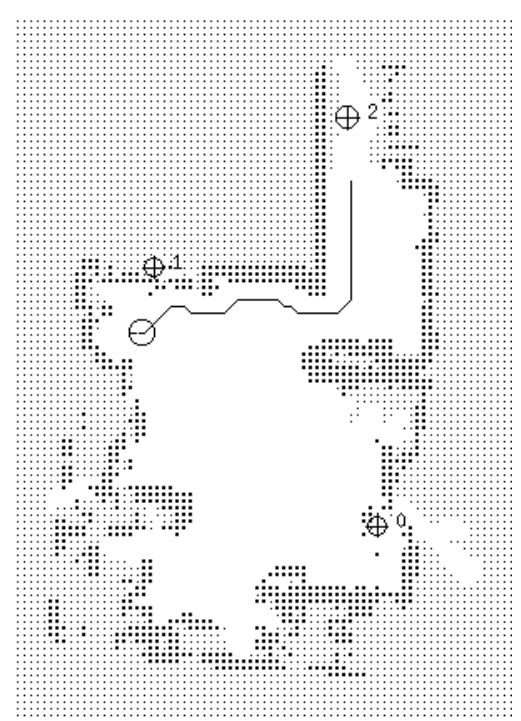

(f)

Figure 2: Frontier-based exploration of an office 
Cells with low occupancy probability are represented by white space; cells with unknown occupancy probability are represented by small dots; and cells with high occupancy probability are represented by large dots. The robot's position is represented by the black circle with a line marking the robot's orientation. The robot's path is indicated by the black line.

In Figure 2a, the robot starts in the center of the hallway, builds an evidence grid using laser-limited sonar, and detects three frontiers. The robot navigates to the closest frontier, Frontier 1. In Figure 2b, the robot has arrived at its destination and added the observations from its new location to the evidence grid. The robot detects two frontiers and navigates to the closest, Frontier 0 . This frontier corresponds to an open doorway leading to an unexplored office. (Frontiers are numbered based on the current grid, so Frontier 2 in Figure $2 \mathrm{a}$ is the same as Frontier 1 in Figure 2b.) In Figure 2c, the robot has moved through the doorway, detecting a new frontier in the center of the room at the boundary of its usable sonar range (ten feet).

Figure $2 \mathrm{~d}$ shows the robot after it has explored more of the office. The robot detects five frontiers in this grid, and it navigates to the closest, Frontier 2. In Figure 2e, the robot detect six frontiers, but the two closest frontiers are inaccessible. Frontier 1 is between a chair and a desk, and Frontier 2 is a narrow gap between two desks. In both cases, there is insufficient clearance to allow the robot to navigate to the frontier, so the robot navigates to the nearest accessible frontier, Frontier 0.

In Figure 2f, the robot has completed its exploration of the office. The total time required was about half an hour. An improved version of this system can map the same office in about fifteen minutes.

The two remaining frontiers are the result of specular reflections from sonar hitting obstacles that are difficult to detect using the laser (from certain angles). Frontier 0 corresponds to a black filing cabinet, and Frontier 1 corresponds to a gray bookshelf. The robot's path planner determines that both of these frontiers are inaccessible, so it plans a path to Frontier 2 in the hallway. The robot then follows this path out of the office to further explore the building.

Frontier-based exploration has also been tested in a large lab/office area. Figure 3 shows the evidence grid constructed during this exploration. The lab area, at the top of the image, contains large open spaces as well as large crates, small boxes, chairs, tables, and bookshelves. The office area, at the bottom of the image, is narrow and cluttered with chairs, desks, and workstations that require precise navigation to avoid collisions with obstacles. Although the total area is larger ( 45 feet $\times 25$ feet), the robot was able to map the open spaces quickly, mapping the entire environment in about half an hour.

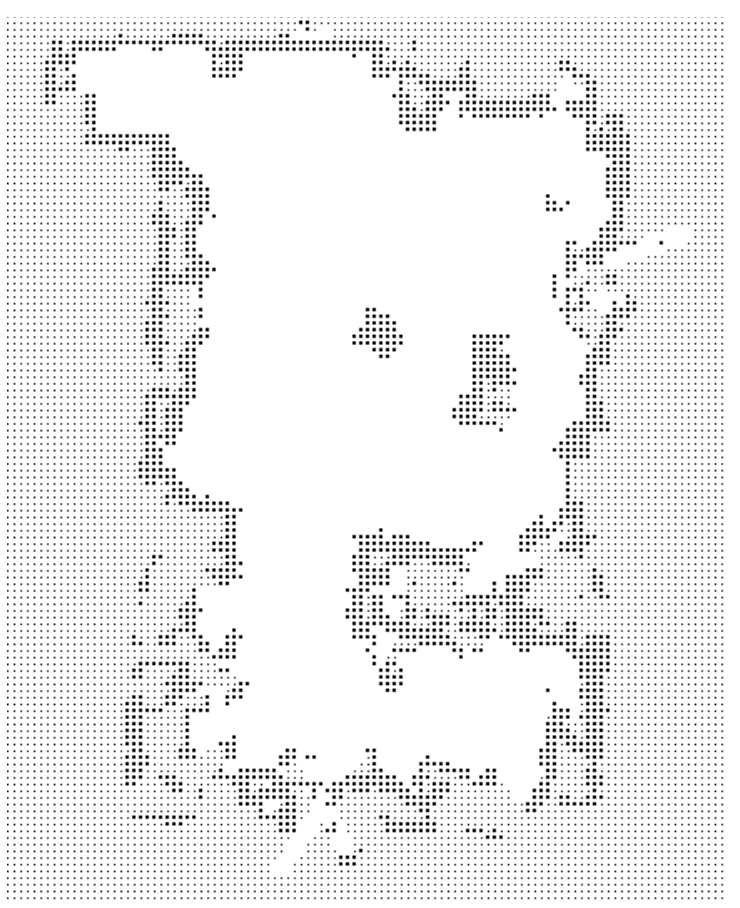

Figure 3: Evidence grid from frontier-based exploration of a large lab/office area

\subsection{Related Work}

Considerable research has been done in robot mapbuilding, but most of this research has been conducted in simulation [4] or with robots that passively observe the world as they are moved by a human controller [2] [3]. However, a few systems for autonomous exploration have been implemented on real robots.

Mataric [6] has developed Toto, a robot that combines reactive exploration, using wall-following and obstacleavoidance, with a simple topological path planner. The reactive nature of Toto's exploration limits its ability to map environments where wall-following is insufficient to explore the complex structure of the world.

We previously developed a reactive/topological exploration system [10] for ELDEN. This system had the advantage of being able to adapt its topological map to changes encountered in the environment. However, it also suffered the limitations of a purely reactive exploration strategy, in terms of the size and complexity of the environments that it could explore efficiently.

Connell [1] has developed a simple exploration system to demonstrate his SSS architecture. This system was limited to mapping hallways where doors and corridors intersect at 90 degree angles.

Lee [5] has implemented Kuipers Spatial Semantic Hierarchy [4] on a real robot. However, this approach also 
assumes that all walls are parallel or perpendicular to each other, and this system has only been tested in a simple environment consisting of a three corridors constructed from cardboard barriers.

Thrun and Bücken [9] have developed an exploration system that builds a spatial representation that combines evidence grids with a topological map. This system has been able to explore the network of hallways within a large building. While this approach works well within the hallway domain, it assumes that all walls are either parallel or perpendicular to each other, and that they do not deviate more than 15 degrees from these orientations. An implicit assumption is that walls are observable and not obstructed by obstacles. These assumptions make this approach unsuitable for rooms cluttered with obstacles that may be in arbitrary orientations.

A robot using frontier-based exploration has three advantages over the systems described above. First, it can explore environments containing both open and cluttered spaces. Second, it can explore environments where walls and obstacles are in arbitrary orientations. Third, it can explore efficiently by moving to the locations that are most likely to add new information to the map.

\subsection{Conclusions and Future Work}

In this paper, we have introduced a new approach to exploration based on the concept of frontiers, regions on the boundary between open space and unexplored space. By constantly moving to new frontiers, a mobile robot can extend its map into new territory until the entire environment has been explored. We have described and implemented a method for detecting frontiers in evidence grids and navigating autonomously to these frontiers. We have also introduced a technique for minimizing specular reflections in evidence grids using laser-limited sonar. We have tested this approach with a real mobile robot, by exploring two different real-world office environments cluttered with a variety of obstacles.

We have recently integrated frontier-based exploration with the continuous localization techniques developed by Schultz, Adams, and Grefenstette [8]. The integrated system is designed to explore large environments while maintaining an accurate position estimate. Initial results have been very promising, and we are currently conducting experiments to test the performance of the integrated system.

\subsection{Acknowledgments}

This work is supported by the Office of Naval Research. Alan Schultz and John Grefenstette provided useful comments on this research.

\subsection{References}

[1] Jonathan Connell and Sridhar Mahadevan, "Rapid task learning for real robots," in Robot Learning, Jon Connell and Sridhar Mahadevan, Eds., Boston, MA: Kluwer Academic, pp. 105-139, 1993.

[2] Sean Engelson, Passive Map Learning and Visual Place Recognition, Ph.D. Thesis, Department of Computer Science, Yale University, 1994.

[3] David Kortenkamp, Cognitive Maps for Mobile Robots: A Representation for Mapping and Navigation, Ph.D. Thesis, Electrical Engineering and Computer Science Department, University of Michigan, 1993.

[4] Benjamin Kuipers and Yung-Tai Byun, "A robot exploration and mapping strategy based on a semantic hierarchy of spatial representations," Journal of Robotics and Autonomous Systems, 8:4763, 1991.

[5] Wan Yik Lee, Spatial Semantic Hierarchy for a Physical Robot, Ph.D. Thesis, Department of Computer Sciences, The University of Texas at Austin, 1996.

[6] Maja Mataric, "Integration of representation into goal-driven behavior-based robots," IEEE Transactions on Robotics and Automation, 8(3):304-312, June 1992.

[7] Hans Moravec and Alberto Elfes, "High resolution maps from wide angle sonar," Proceedings of the IEEE International Conference on Robotics and Automation, St. Louis, MO, 1985, pp. 116-121.

[8] Alan Schultz, William Adams, and John Grefenstette, "Continuous localization using evidence grids," NCARAI Technical Report AIC96-007, Naval Research Laboratory, Washington, DC, 1997.

[9] Sebastian Thrun and Arno Bücken, "Integrating grid-based and topological maps for mobile robot navigation," Proceedings of the Thirteenth National Conference on Artificial Intelligence (AAAI-96), Portland, OR, August 1996, pp. 944-950.

[10] Brian Yamauchi and Randall Beer, "Spatial learning for navigation in dynamic environments," IEEE Transactions on Systems, Man, and Cybernetics Part B: Cybernetics, Special Issue on Learning Autonomous Robots, 26(3):496-505, June 1996. 